\title{
Eficiencia del método LDG para aproximar la solución de los problemas de Bratu y de Troesch
}

\author{
Paul E. Castillo ${ }^{1}$ y Sergio A. Gómez ${ }^{2}$ \\ ${ }^{1}$ Departamento de Ciencias Matemáticas, Universidad de Puerto Rico, Mayagüez, mail: paul.castillo@upr.edu \\ ${ }^{2}$ Departamento de Ciencias Matemáticas, Universidad de Puerto Rico, Mayagüez, mail: sergio.gomez@upr.edu
}

Recibido: 31 de Mayo de 2017 / Aceptado: 21 de Agosto de 2017

\begin{abstract}
Resumen
Se realiza un estudio numérico del método de elemento finito "Local Discontinuous Galerkin" (LDG) aplicado a los problemas no lineales de Bratu y de Troesch en el régimen estacionario. A diferencia de otros esquemas numéricos, se muestra la capacidad del método para a) aproximar ambas soluciones de bifurcación en el problema de Bratu; y b) obtener soluciones para valores grandes del parámetro de Troesch. Además se muestra la ventaja de utilizar polinomios de alto orden para obtener aproximaciones muy precisas.
\end{abstract}

Palabras clave: Problema de Bratu y de Troesch, ecuación de difusión y reacción no lineal, método Local Discontinuous Galerkin ( $L D G)$, aproximaciones de elemento finito de alto orden.

\begin{abstract}
A numerical study of the finite element method "Local Discontinuous Galerkin" (LDG) applied to the non-linear Bratu's and Troesch's problem in the steady state regime is presented. Unlike other numerical schemes, it is shown, numerically, the ability of the LDG method a) to approximate both bifurcation solutions in Bratu's problem; and b) to obtain solutions for large values of Troesch's parameter. The advantage of using high-order polynomials to obtain accurate approximations is also addressed.
\end{abstract}

Keywords: Bratu and Troesch problems, diffusion and non linear reaction equation, Local Discontinuous Galerkin method (LDG), high order finite element approximations.

PACS: 02.60.Cb; 02.70.Dh; 89.20.-a; 89.20.Kk

\section{INTRODUCCIÓN}

$\mathrm{D}$ IVERSOS fenómenos físicos se modelan matemáticamente mediante una ecuación en derivadas parciales conocida como ecuación de difusión-reacción. Concretamente en $[a, b]$ y condiciones de Dirichlet, $u(a)=$ $u_{a}$ y $u(b)=u_{b}$ :

$$
-\left(\kappa u^{\prime}\right)^{\prime}+r(u)=f, \quad \text { en }(a, b),
$$

donde se asume la existencia de $\kappa_{0}>0$ tal que $0<\kappa_{0} \leq$ $\kappa(x)$ para todo $x \in(a, b) ;$ y $r: \mathbb{R} \longrightarrow \mathbb{R}$ es una función no lineal. Debido a la naturaleza no lineal del término de reacción, resulta difícil, y en algunos casos imposible, encontrar una expresión analítica para la solución de dichos problemas; por lo que se debe recurrir a métodos numéricos para su aproximación. En este trabajo nos concentramos particularmente en la aproximación numérica de dos problemas cuya formulación se describe mediante la ecuación modelo (1):

a) El problema de Bratu, 4, el cual se utiliza como modelo de ignición de combustible, se describe como un problema no lineal de valores propios, con $u(0)=$ $0, u(1)=0$ y cuya forma es la siguiente

$$
-u^{\prime \prime}+\lambda e^{u}=0, \text { en }(0,1)
$$

La variable $u$ representa un exceso de temperatura adimensional de un material combustible, el parámetro $\lambda$ es una tasa adimensional de producción de calor. Para reacciones químicas en un material rígido, el término exponencial representa el calor generado por la reacción química mientras que el operador diferencial de segundo orden modela el calor removido por conducción. Este modelo también se utiliza en áreas tales como nanotecnología, [1]; transferencia de calor radiactivo, [3]; teoría de reactores químicos, [11] teoría de reacciones térmicas [13]; y el modelo de Chandrasekhar para la expansión del universo [14, 22]. Asimismo, este ha sido ampliamente utilizado como punto de referencia para validar propiedades de los métodos numéricos: tasa de convergencia, precisión y estabilidad. En [13 puede encontrarse una descripción detallada acerca de la historia e 
importancia de este problema.

b) El problema de Troesch [21], el cual surge de la investigación del confinamiento de una columna de plasma por una presión de radiación [23] y también en la teoría de electrodos de gas poroso [12, 17], es expresado como $u(0)=0, u(1)=1$ :

$$
-u^{\prime \prime}+\beta \sinh \beta u=0, \text { en }(0,1),
$$

donde $\beta$ se conoce como el parámetro de Troesch. En [21], se demostró que este problema tiene una singularidad aproximadamente en $x_{s}=\frac{1}{\beta} \ln \left(\frac{8}{y^{\prime}(0)}\right)$; lo cual dificulta la aproximación numérica para valores grandes de este parámetro.

Se debe señalar que existen expresiones explícitas para la solución de ambos problemas; Ascher presenta una para el problema de Bratu en [2]; Roberts y Shipman proponen una para la ecuación de Troesch en [20. Ambas expresiones requieren del conocimiento de un punto fijo específico; sin embargo, el cálculo de dicho punto, es por lo general un gran reto numérico para cierto rango de valores característicos del problema; de ahí el interés en el desarrollo de discretizaciones espaciales que puedan ser exitósamente aplicadas a la ecuación original.

El método de elemento finito Galerkin Discontinuo es una técnica de discretización espacial que ha dejado de ser un método experimental; y es considerada en la actualidad un método muy versátil para aproximar ecuaciones en derivadas parciales. Uno de estos métodos es el "Local Discontinuous Galerkin" (LDG por sus siglas en inglés), el cual fue propuesto originalmente por Cockburn and Shu, en [10]. Debido a sus excelentes propiedades de estabilidad y alta precisión, este ha sido aplicado a una gran variedad de ecuaciones. El primer análisis de convergencia del método LDG para un problema de difusión pura, $(r(\cdot) \equiv 0)$ se presentó en Castillo y col. [7]; Perugia y Schötzau analizaron el método para la versión $h p$ en [19]; y un resultado de superconvergencia para la variable auxiliar se discutió en Cockburn y col. en [9]. En [5], Castillo realizó un estudio comparativo, el cual reveló cierta superioridad del método LDG en comparación a otros métodos discontinuos similares. El presente estudio es el primero en considerar el método LDG, en su versión estabilizada, para la aproximación de estos problemas.

\section{MÉtodo LDG EN 1D}

A continuación se describe, brevemente, la formulación del método LDG para el problema modelo de difusión y reacción no lineal, Ecn. (1). Para ello, se procede al igual que en [7], reescribiendo el problema modelo como un sistema de primer orden en las variables $(\boldsymbol{q}, u)$, donde la variable auxiliar $\boldsymbol{q}=-\kappa u^{\prime}$, se obtiene

$$
\begin{aligned}
\kappa^{-1} \boldsymbol{q}+u^{\prime} & =0 \\
\boldsymbol{q}^{\prime}+r(u) & =f
\end{aligned}
$$

Sea $\mathcal{T}_{h}=\left\{x_{k}\right\}_{k=0}^{N}$ una partición de $[a, b]$, con puntos no necesariamente uniformemente distribuidos; en cada celda $I_{k}=\left(x_{k}, x_{k+1}\right)$, el método LDG, busca aproximaciones $u_{h}(\cdot)$ y $\boldsymbol{q}_{h}(\cdot)$ de $u(\cdot)$ y $\boldsymbol{q}(\cdot)$ respectivamente; en el espacio, $\mathbb{P}_{p_{k}}$, de polinomios de grado menor o igual a $p_{k}$, con $p_{k} \geq 1$; de tal manera que las siguientes ecuaciones se verifiquen para toda función de prueba $\boldsymbol{r}, v \in \mathbb{P}_{p_{k}}$

$$
\begin{aligned}
\int_{I_{k}} \kappa^{-1} \boldsymbol{q}_{h} \cdot \boldsymbol{r}+\left.\widehat{u_{h}} \boldsymbol{r}\right|_{x_{k}} ^{x_{k+1}}-\int_{I_{k}} u_{h} \boldsymbol{r}^{\prime} & =0 \\
\left.v \widehat{\boldsymbol{q}_{h}}\right|_{x_{k}} ^{x_{k+1}}-\int_{I_{k}} \boldsymbol{q}_{h} v^{\prime}+\left.v s\left(u_{h}\right)\right|_{x_{k}} ^{x_{k+1}}+\int_{I_{k}} r\left(u_{h}\right) v & =\int_{I_{k}} v f
\end{aligned}
$$

Nótese que la continuidad de la aproximación en los nodos $x_{k}$ se impone débilmente por medio de flujos numéri$\cos , \widehat{u_{h}} \mathrm{y} \widehat{\boldsymbol{q}_{h}}$, que no son más que aproximaciones de $u$ y $\boldsymbol{q}$, en cada nodo. Estos se definen de manera general, para todo nodo interior $x_{k}$, como combinaciones convexas de los valores de $\boldsymbol{q}_{h}$ y $u_{h}$ en las celdas $I_{k-1}$ e $I_{k}$; específicamente, sea $\lambda_{k} \in[0,1]$, el cual llamaremos parámetro de gradiente del método LDG:

$$
\begin{aligned}
& \widehat{u_{h}}\left(x_{k}\right)=\left(1-\lambda_{k}\right) u_{h}^{k-1}\left(x_{k}^{-}\right)+\lambda_{k} u_{h}^{k}\left(x_{k}^{+}\right) \\
& \widehat{\boldsymbol{q}_{h}}\left(x_{k}\right)=\lambda_{k} \boldsymbol{q}_{h}^{k-1}\left(x_{k}^{-}\right)+\left(1-\lambda_{k}\right) \boldsymbol{q}_{h}^{k}\left(x_{k}^{+}\right)
\end{aligned}
$$

En los nodos de frontera, se definen utilizando el dato de Dirichlet; es decir,

$$
\begin{array}{ll}
\widehat{u_{h}}\left(x_{0}\right)=u_{a} & \widehat{u_{h}}\left(x_{N}\right)=u_{b} \\
\widehat{\boldsymbol{q}_{h}}\left(x_{0}\right)=\boldsymbol{q}_{h}\left(x_{0}^{+}\right) & \widehat{\widehat{\boldsymbol{q}_{h}}}\left(x_{N}\right)=\boldsymbol{q}\left(x_{N}^{-}\right)
\end{array}
$$

La influencia del parámetro $\lambda_{k}$ se discutió en [9] para mallas Cartesianas y en [6] para el caso general de mallas no estructuradas. El término de estabilidad $s(\cdot)$, el cual garantiza la existencia y unicidad de la solución del problema discreto, [7, 19, en el caso de difusión pura (sin reacción) se define por

$$
s\left(u_{h}\right)\left(x_{k}\right)=\frac{\eta_{k}}{h_{k}}\left(u_{h}^{k}\left(x_{k}^{+}\right)-u_{h}^{k-1}\left(x_{k}^{-}\right)\right)
$$

donde $\eta_{k}>0$, y $h_{k}=\operatorname{máx}\left\{l\left(I_{k-1}\right), l\left(I_{k}\right)\right\}$ para todo $k=1, \ldots, N-1$. Finalmente, sumando sobre todas las celdas, se obtiene un sistema no lineal de la forma

$$
\begin{aligned}
D \boldsymbol{q}_{h}+B u_{h} & =b_{q} \\
-B^{T} \boldsymbol{q}_{h}+S u_{h}+\mathcal{R}_{h}\left(u_{h}\right) & =b_{u}
\end{aligned}
$$

donde las matrices $D, B$ y $S$ son los operadores discretos 
de difusión lineal, gradiente y estabilidad, respectivamente; y $\mathcal{R}_{h}(\cdot)$ es el operador discreto de reacción no lineal.

\section{A. Detalles sobre implementación.}

Nótese que al no imponer continuidad, la matriz de difusión $D$ es diagonal por bloques; por lo que en la práctica, el sistema no lineal, dado por las ecuaciones (9a) y $(9 \mathrm{~b})$, se reduce a un sistema no lineal en la variable primaria $u_{h}$ :

$$
\mathcal{F}_{h}\left(u_{h}\right):=\mathcal{A}_{h} u_{h}+\mathcal{R}_{h}\left(u_{h}\right)-b=0
$$

donde la matriz $\mathcal{A}_{h}=S+B^{T} D^{-1} B$ representa la discretización del operador, $-\Delta(\cdot)$ y $b=b_{u}-B^{T} D^{-1} b_{q}$. En [8, Castillo y Sequeira, presentaron un algoritmo de ensamblado rápido y eficiente para la matriz $\mathcal{A}_{h}$, el cual no requiere del almacenamiento de las matrices globales $D, B, S$; dicho procedimiento es de gran interés para simulaciones en 2D y 3D.

El sistema no lineal global se resuelve mediante la iteración de Newton para sistemas, la cual se implementa de la siguiente forma, para $l=0,1, \ldots$ :

$$
\begin{aligned}
\delta^{(l)} & =u^{(l+1)}-u^{(l)} \\
\left(D \mathcal{F}_{h}\left(u^{(l)}\right)\right) \delta^{(l)} & =-\mathcal{F}_{h}\left(u^{(l)}\right) \\
u^{(l+1)} & =u^{(l)}+\delta^{(l)}
\end{aligned}
$$

Puesto que la dimensión del dominio espacial es 1D; en nuestras simulaciones, el sistema lineal Ecn. (11b), no es de gran tamaño, no más de veinte mil incógnitas, por lo que este se resuelve simplemente por el método de eliminación de Gauss; obviamente, para problemas en 2D/3D, es más conveniente utilizar un método iterativo como Gradiente Conjugado, GMRES, BiCG o QMR. Esto se tratará en un próximo trabajo.

Esencialmente, la iteración de Newton requiere dos operaciones: a) la evaluación del operador no lineal $\mathcal{F}_{h}(\cdot)$; y, b) la generación del Jacobiano $D \mathcal{F}_{h}(\cdot)$. A continuación describimos en detalle el cálculo de estos operadores. Sin pérdida de generalidad, se asume aproximaciones de grado uniforme $p$ y como base local para el espacio de elemento finito $\mathbb{P}_{k}$, en la celda de referencia $\hat{I}=[-1,1]$, se considera la familia de polinomios ortogonales de Legendre $\left\{L_{j}(\hat{x})\right\}_{j=0}^{p}$. La base correspondiente a la celda $I_{k}$, mediante el mapeo local $\psi_{k}: \hat{I} \longrightarrow I_{k}$, se reduce a $\left\{\phi_{j}=L_{j} \circ \psi_{k}^{-1}\right\}_{j=0}^{p} ;$ y la aproximación $u_{h}^{k}(x)$ en esa celda, se escribe como $u_{h}^{k}(x)=\sum_{j=0}^{p} U_{j}^{k} \phi_{j}(x)$.

A diferencia del método de elemento finito tradicional; el cual impone, fuertemente, la continuidad entre celdas adyacentes; en los métodos discontinuos, el término de reacción se calcula; de manera independiente, celda por celda, y contribuye específicamente a la parte del vector global asociado a esa celda. Sea $\left\{\hat{x}_{l}, \omega_{l}\right\}_{l=0}^{N}$ una cuadratura suficientemente precisa. La contribución de la celda $I_{k}$ al término de reacción, vector $\left[\mathcal{R}_{h}\left(u_{h}\right)\right]^{k}$, se aproxima, numéricamente de la siguiente manera. Para todo $i=$ $0, \ldots, p$ :

$$
\left[\mathcal{R}_{h}\left(u_{h}\right)\right]_{i}^{k}=\int_{I_{k}} r\left(u_{h}^{k}(x)\right) \phi_{i}(x) d x \approx\left|D \psi_{k}\right| \sum_{l=0}^{N} \omega_{l} r\left(u_{h}^{k} \circ \psi_{k}\left(\hat{x}_{l}\right)\right) L_{i}\left(\hat{x}_{l}\right)
$$

El cálculo de $D \mathcal{F}_{h}\left(u_{h}\right)=\mathcal{A}_{h}+D \mathcal{R}_{h}\left(u_{h}\right)$, se reduce al Jacobiano de la parte no lineal, $D \mathcal{R}_{h}\left(u_{h}\right)$. Además es importante resaltar que debido a la naturaleza del método; este se calcula; una vez más de manera independiente, celda por celda. La matriz resultante es diagonal por

$$
\left[D \mathcal{R}_{h}\left(u_{h}\right)\right]_{i j}^{k}=\int_{I_{k}} \operatorname{Dr}\left(u_{h}^{k}(x)\right) \phi_{i}(x) \phi_{j}(x) d x \approx\left|D \psi_{k}\right| \sum_{l=0}^{N} \omega_{l} \operatorname{Dr}\left(u_{h}^{k} \circ \psi_{k}\left(\hat{x}_{l}\right)\right) L_{i}\left(\hat{x}_{l}\right) L_{j}\left(\hat{x}_{l}\right)
$$

En la Figura 1 se muestra la estructura de las matrices $\mathcal{A}_{h}$ y $D \mathcal{R}_{h}\left(u_{h}\right)$; las cuales son matrices huecas por bloques.

bloques; donde cada bloque es de tamaño $p+1 \times p+1$. Para todo $i, j=0, \ldots, p$, la entrada $(i, j)$ del bloque $\left[D \mathcal{R}_{h}\left(u_{h}\right)\right]^{k}$, asociado a la celda $I_{k}$, se aproxima de la siguiente manera:

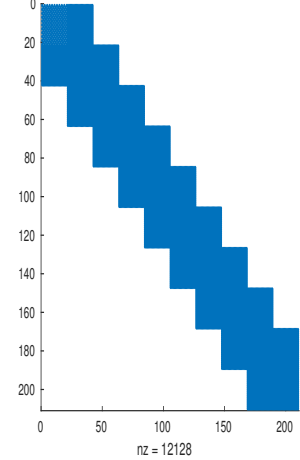

(a) Parte difusiva: $\mathcal{A}_{h}$

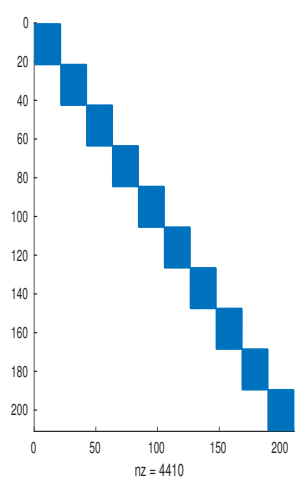

(b) Parte reactiva: $D \mathcal{R}_{h}\left(u_{h}\right)$

Figura 1: Patrón de matrices en $D \mathcal{F}_{h}(\cdot)$. 


\section{EXPERIMENTOS NUMÉRICOS}

Los siguientes experimentos muestran la eficiencia del método LDG para resolver los retos particulares de los problemas de Bratu y de Troesch. La tolerancia utilizada en cada experimento para el método de Newton fue $t o l=$ $10^{-14}$; y se usó el error relativo de dos aproximaciones consecutivas: $\left\|u_{h}^{n+1}-u_{h}^{n}\right\|_{2}<\left\|u_{h}^{n}\right\|_{2} *$ tol como criterio de parada.

\section{A. Problema de Bratu}

El principal resultado sobre la existencia y multiplicidad de soluciones del problema de Bratu, [4, muestra que existe un valor crítico $\lambda_{c} \approx 3.513830719$ (parámetro de Kamenetskii) para el cual, la ecuación posee 2 soluciones si $\lambda<\lambda_{c}$, una para $\lambda=\lambda_{c}$ y ninguna para $\lambda>\lambda_{c}$.

Como primer objetivo deseamos mostrar que el método LDG converge de manera óptima en $h$ para el potencial $u_{h}$; es decir orden $\mathcal{O}\left(h^{p+1}\right)$ donde $h$ está definida por, $h=\operatorname{máx}\left\{l\left(I_{k}\right): k=0, \ldots, N-1\right\} ;$ y $\mathcal{O}\left(h^{p}\right)$ para el gradiente, $\boldsymbol{q}_{h}$. Para ello comparamos los resultados obtenidos con la solución exacta presentada en [2], la cual está dada por

$$
u(x)=-2 \ln \left[\frac{\cosh \left(\left(x-\frac{1}{2}\right) \frac{\theta}{2}\right)}{\cosh \left(\frac{\theta}{2}\right)}\right],
$$

donde $\theta$ resuelve

$$
\theta=\sqrt{2 \lambda} \cosh \left(\frac{\theta}{4}\right) .
$$

Las Tablas 11 y 2 muestran las tasas de convergencia del error en la norma $L_{2}$ para aproximaciones de grado $p=1,2$ y $3 ;$ con $\lambda=1$. La malla inicial es de 10 celdas con puntos uniformemente distribuidos, y las siguientes fueron obtenidas por refinamiento uniforme.

Como segundo objetivo se muestra que el método LDG es capaz de encontrar ambas soluciones para el caso $\lambda<\lambda_{c}$, lo cual no es posible para otros esquemas numéricos: como el método de diferencias finitas estándar, y el método de descomposición de Adomian [18. Las Figuras 2 , 3 y 4 muestran las gráficas de la solución exacta y aproximada; para ambas ramas de bifurcación, y para $\lambda=3.0,3.5,3.513$, respectivamente. Nótese que a medida $\lambda$ se aproxima al valor crítico $\lambda_{c}$, ambas soluciones se aproximan; lo cual es de esperar ya que para dicho valor, el problema posee exactamente una solución.

Los cálculos se realizaron con una malla uniforme de 20 celdas y polinomios de grado $p=1$. Como aproximación inicial para el método de Newton, se utilizó $u_{h}^{(0)}(x)=\alpha \sin (\pi x)$, como se sugiere en [15]; en este caso se utilizó $\alpha=1,3$ para la solución inferior y superior respectivamente.

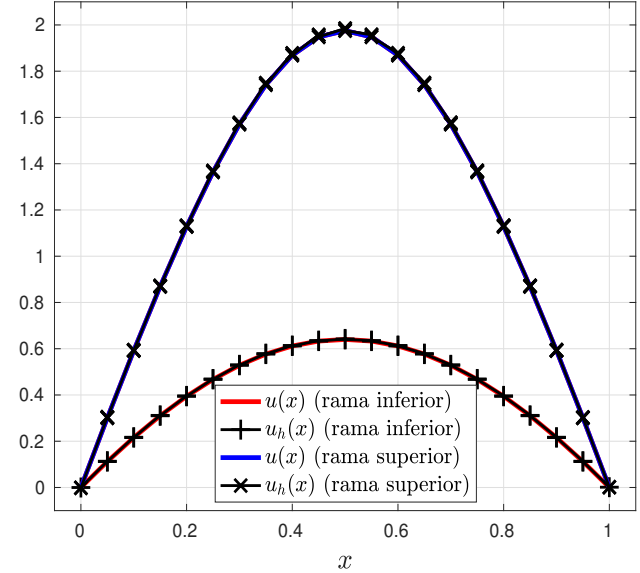

Figura 2: Aproximación del problema de Bratu, $\lambda=3.0$.

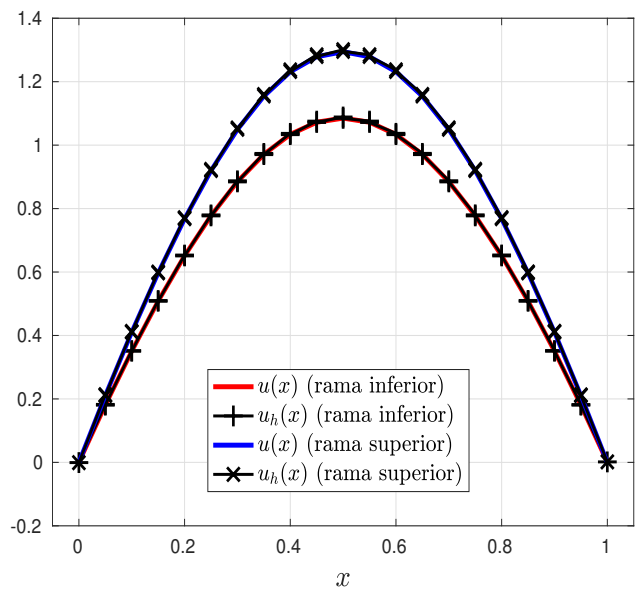

Figura 3: Aproximación del problema de Bratu, $\lambda=3.5$.

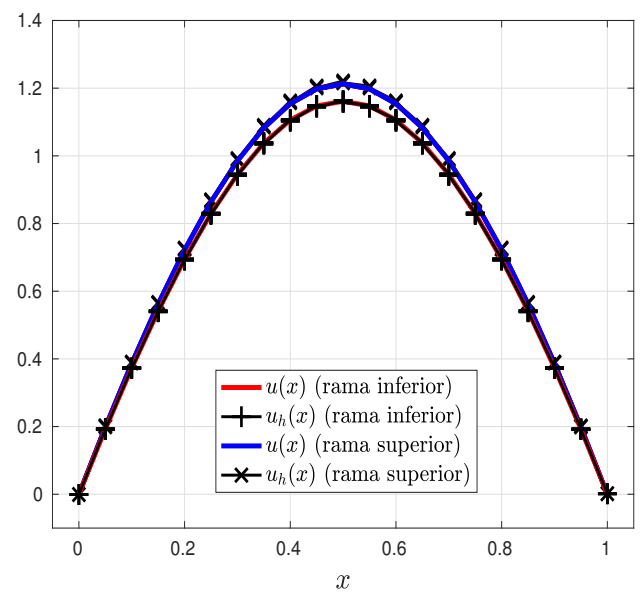

Figura 4: Aproximación del problema de Bratu, $\lambda=3.513$.

\section{B. Problema de Troesch}

$\mathrm{Al}$ igual que en el problema anterior, nuestro primer objetivo es mostrar que el método converge de manera 


\begin{tabular}{|c|cc|cc|cc|}
\hline \multirow{2}{*}{$h$} & \multicolumn{2}{|c|}{$p=1$} & \multicolumn{2}{c|}{$p=2$} & \multicolumn{2}{c|}{$p=3$} \\
\cline { 2 - 7 } & Error & Tasa & Error & Tasa & Error & Tasa \\
\hline \hline $1.00 \mathrm{e}-01$ & $6.44 \mathrm{e}-04$ & - & $1.80 \mathrm{e}-06$ & - & $3.56 \mathrm{e}-08$ & - \\
$5.00 \mathrm{e}-02$ & $1.62 \mathrm{e}-04$ & $1.99 \mathrm{e}+00$ & $2.03 \mathrm{e}-07$ & $3.15 \mathrm{e}+00$ & $2.09 \mathrm{e}-09$ & $4.09 \mathrm{e}+00$ \\
$2.50 \mathrm{e}-02$ & $4.08 \mathrm{e}-05$ & $1.99 \mathrm{e}+00$ & $2.54 \mathrm{e}-08$ & $3.00 \mathrm{e}+00$ & $1.29 \mathrm{e}-10$ & $4.01 \mathrm{e}+00$ \\
$1.25 \mathrm{e}-02$ & $1.02 \mathrm{e}-05$ & $2.00 \mathrm{e}+00$ & $3.20 \mathrm{e}-09$ & $2.99 \mathrm{e}+00$ & $8.09 \mathrm{e}-12$ & $4.00 \mathrm{e}+00$ \\
$6.25 \mathrm{e}-03$ & $2.56 \mathrm{e}-06$ & $2.00 \mathrm{e}+00$ & $4.01 \mathrm{e}-10$ & $2.99 \mathrm{e}+00$ & $5.41 \mathrm{e}-13$ & $3.90 \mathrm{e}+00$ \\
\hline \hline
\end{tabular}

Tabla 1: Convergencia del potencial $u_{h}$ para $\lambda_{k}=0$

\begin{tabular}{|c|cc|cc|cc|}
\hline \multirow{2}{*}{$h$} & \multicolumn{2}{|c|}{$p=1$} & \multicolumn{2}{c|}{$p=2$} & \multicolumn{2}{c|}{$p=3$} \\
\cline { 2 - 7 } & Error & Tasa & Error & Tasa & Error & Tasa \\
\hline \hline $1.00 \mathrm{e}-01$ & $1.03 \mathrm{e}-03$ & - & $5.59 \mathrm{e}-06$ & - & $6.50 \mathrm{e}-08$ & - \\
$5.00 \mathrm{e}-02$ & $5.09 \mathrm{e}-04$ & $1.02 \mathrm{e}+00$ & $8.78 \mathrm{e}-07$ & $2.67 \mathrm{e}+00$ & $6.73 \mathrm{e}-09$ & $3.27 \mathrm{e}+00$ \\
$2.50 \mathrm{e}-02$ & $2.55 \mathrm{e}-04$ & $9.99 \mathrm{e}-01$ & $1.73 \mathrm{e}-07$ & $2.35 \mathrm{e}+00$ & $7.90 \mathrm{e}-10$ & $3.09 \mathrm{e}+00$ \\
$1.25 \mathrm{e}-02$ & $1.28 \mathrm{e}-04$ & $9.97 \mathrm{e}-01$ & $3.99 \mathrm{e}-08$ & $2.11 \mathrm{e}+00$ & $9.71 \mathrm{e}-11$ & $3.02 \mathrm{e}+00$ \\
$6.25 \mathrm{e}-03$ & $6.39 \mathrm{e}-05$ & $9.98 \mathrm{e}-01$ & $9.80 \mathrm{e}-09$ & $2.03 \mathrm{e}+00$ & $1.21 \mathrm{e}-11$ & $3.00 \mathrm{e}+00$ \\
\hline \hline
\end{tabular}

Tabla 2: Convergencia de la variable auxiliar $\boldsymbol{q}_{h}$ para $\lambda_{k}=0$.

óptima en $h$. Para ello comparamos los resultados obtenidos con la solución exacta presentada en [21]:

$$
u(x)=\frac{2}{\beta} \sinh ^{-1}\left(\frac{a}{2} s c(\beta x, m)\right),
$$

donde $s c(\cdot, \cdot)$ es la función elíptica de Jacobi, $a=2(1-m)^{1 / 2}$ y $m$ resuelve

$$
\frac{\sinh \left(\frac{\beta}{2}\right)}{(1-m)^{1 / 2}}=s c(\beta, m) .
$$

Es importante resaltar que aún teniendo una expresión analítica para la solución, es extremadamente difícil obtener, con suficiente precisión, el valor de $m$ apropiado para valores grandes de $\beta$. De acuerdo al análisis presentado por Roberts y Shipman en [20, la dificultad estriba en el cálculo de la solución de interés de la ecuación (17), la cual posee múltiples soluciones que se acumulan en 1 . Esto se ilustra en la Figura 5

En la Figura 6 se muestran las gráficas de los errores obtenidos por el método LDG, con flujo bidireccional, para $\beta=10$ y 15 , donde se observa un comportamiento asintótico con una tasa de convergencia óptima en $h: \mathcal{O}\left(h^{p+1}\right)$ para el potencial (o variable primaria). Los cálculos se realizaron a partir de una malla inicial de 16 celdas con una distribución geométrica en los nodos y 5 refinamientos globales; y como aproximación inicial se consideró $u_{h}^{(0)}=0$

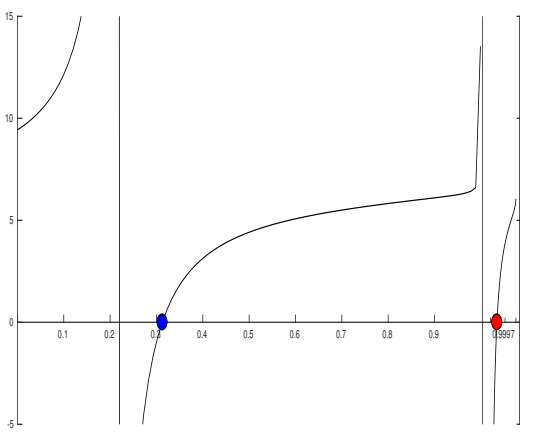

(a) $\beta=5$.

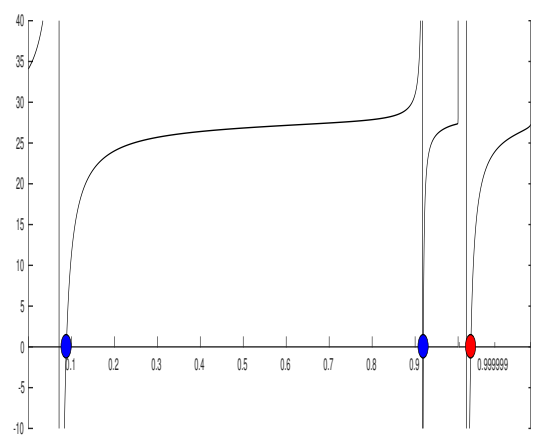

(b) $\beta=10$.

Figura 5: Distribución de los puntos fijos de la ecuación (17). 


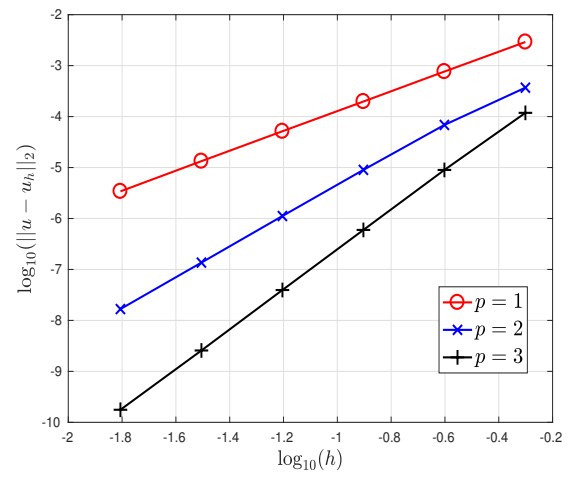

(a) tasas $1.95(p=1)$, 2.92 $(p=2), 3.89(p=3)$.

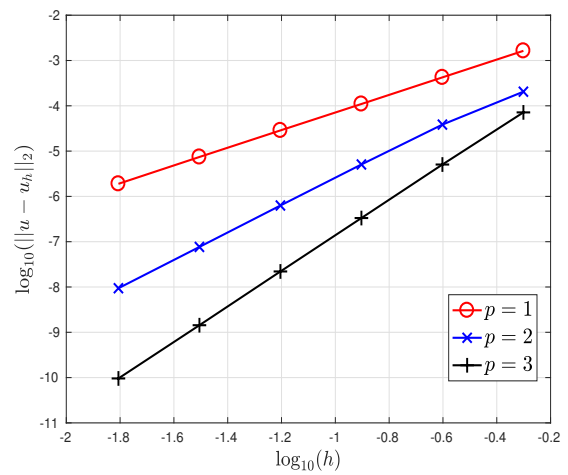

(b) tasas $1.95(p=1), 2.91(p=2), 3.91(p=3)$.

Figura 6: Convergencia del potencial para $\beta=106 a$ y para $\beta=15(6 b)$.

En la Tabla 3 se realiza una comparación con la solución provista por Scott en [16], para algunos puntos particulares; en el caso $\beta=10, p=2 \beta=20$ y $h=0.01$. Se incluye el error relativo porcentual.

El comportamiento del error para el potencial con respecto al grado de aproximación, $p$, se ilustra en la Figura 7, para $\beta=1.5$ y una malla uniforme fija con 5 celdas. La Tabla 4 muestra la cantidad de celdas y grados de libertad que requiere una aproximación de grado 1 para obtener aproximadamente la misma precisión que la obtenida con polinomios de grado $p>1$. Por ejemplo, para obtener una precisión similar a la de $p=6,(35$ grados de libertad), se requiere una malla de 10500 celdas (21000 grados de libertad). Debido a la regularidad de la solución, esto muestra que es más conveniente utilizar aproximaciones de alto orden. Por tal motivo, en la Figura 8 se muestran aproximaciones obtenidas por el método LDG para distintos valores de $\beta$ utilizando $p=2 \beta$ y una malla uniforme de 100 celdas. Nótese que se han incluido valores del parámetro de Troesch para los cuales no se puede usar la expresión analítica, Ecn. (16), debido a la dificultad en el cálculo del punto fijo, $m$, en la ecuación (17).

\begin{tabular}{|r|r|r|r|}
\hline$p$ & Error(1) & \# celdas & \# grados de libertad \\
\hline \hline 2 & $1.4 \mathrm{e}-04$ & 25 & $50(\mathbf{1 5})$ \\
4 & $2.7 \mathrm{e}-07$ & 550 & $1100(\mathbf{2 5})$ \\
6 & $8.9 \mathrm{e}-10$ & 10500 & $21000(\mathbf{3 5})$ \\
8 & $2.6 \mathrm{e}-12$ & 14000 & $28000(\mathbf{4 5})$ \\
\hline
\end{tabular}

Tabla 4: Aproximaciones de grado 1, en negrita grados de libertad para aproximaciones de grado p para una malla de 5 celdas.

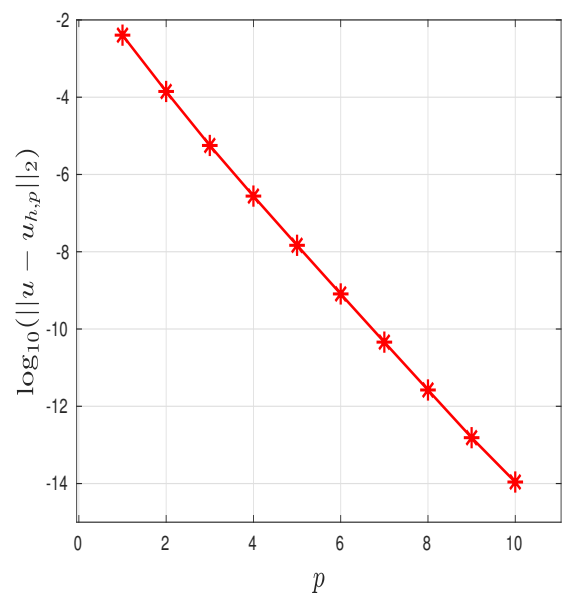

Figura 7: Error del potencial vs p para el problema de Troesch, $\beta=1.5$.

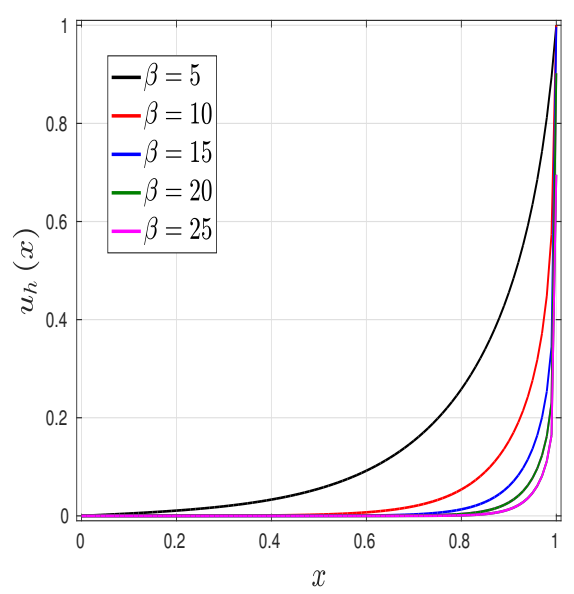

Figura 8: Aproximaciones obtenidas por el método LDG para diferentes valores de $\beta$.

Finalmente, consideramos importante ilustrar, mediante el siguiente ejemplo, la sensibilidad del cálculo de $m$, Ecn. (17), para determinar las tasas de convergencia de un esquema numérico arbitrario; en nuestro caso particular para el LDG. En la Figura 9 se muestra el cálculo del error $L_{2}$ del potencial utilizando aproximaciones de grado 2; $\beta=10$; para 4 representaciones decimales de $m$ con distinta precisión; 5,7,9 y 11 decimales. En principio, se debería obtener la misma línea cuya pendiente indicaría el orden de convergencia del método. Sin embargo, nótese 


\begin{tabular}{|c|c|c|c|}
\hline$x$ & $u(x)[16]$ & $u_{h}(x)$ & $\%$ Error relativo \\
\hline \hline 0 & 0.0000000000000 & 0.0000000000000 & - \\
0.1 & 0.0000421118367 & 0.0000421118993 & $1.49 \mathrm{e}-06$ \\
0.2 & 0.0001299639238 & 0.0001299641158 & $1.48 \mathrm{e}-06$ \\
0.3 & 0.0003589778855 & 0.0003589784014 & $1.44 \mathrm{e}-06$ \\
0.4 & 0.0009779014227 & 0.0009779027719 & $1.38 \mathrm{e}-06$ \\
0.5 & 0.0026590171780 & 0.0026590204905 & $1.25 \mathrm{e}-06$ \\
0.6 & 0.0072289246952 & 0.0072289312132 & $9.02 \mathrm{e}-07$ \\
0.7 & 0.0196640602566 & 0.0196640630977 & $1.44 \mathrm{e}-07$ \\
0.8 & 0.0537303295856 & 0.0537303293505 & $4.38 \mathrm{e}-09$ \\
0.9 & 0.1521140787863 & 0.1521140764058 & $1.56 \mathrm{e}-08$ \\
1.0 & 1.0000000000000 & 0.9999999995951 & $4.05 \mathrm{e}-10$ \\
\hline
\end{tabular}

Tabla 3: Solución puntual del problema de Troesch con el método $L D G$ para $\beta=10$.

que el valor de la solución exacta es extremadamente sensible a la representación decimal de $m$; lo cual se refleja en las pendientes de cada curva; siendo la correcta la obtenida para 11 cifras.

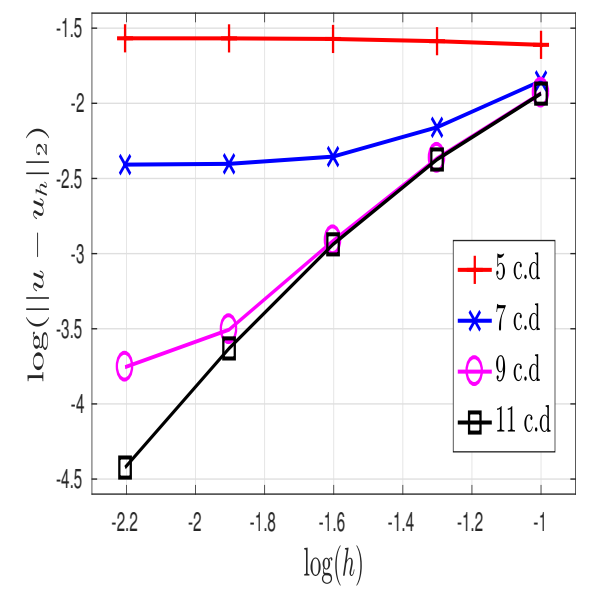

Figura 9: Sensibilidad del cálculo de $m$ para la determinación de las tasas de convergencia.

\section{Conclusiones}

Se presentaron algunos detalles generales sobre la implementación del método Local Discontinuous Galerkin para el estado estacionario de un problema de difusión con reacción no lineal en 1D. En particular se enfatizó sobre el cálculo del término de reacción no lineal y su Jacobiano. Los experimentos numéricos presentados muestran la efectividad del método LDG para los problemas de Bratu y de Troesch, para diferentes valores de los parámetros característicos de dichos problemas: $\lambda$ y $\beta$, respectivamente. Además de obtener aproximaciones con alta precisión, resulta interesante observar, que a pesar de tener una expresión analítica para el problema de Troesch, la cual se reduce, esencialmente, al cálculo de un punto fijo particular, el método LDG es capaz de eludir la dificultad de cálcular dicho punto para grandes valores del parámetro de Troesch. Utilizando soluciones de referencia en ambos problemas, se mostró que el método LDG converge con orden óptimo en $h: \mathcal{O}\left(h^{p+1}\right)$ para el potencial; y orden $\mathcal{O}\left(h^{p}\right)$ para la derivada. De esta manera, se muestra que el método LDG, es un método confiable y robusto, que puede ser utilizado para obtener soluciones en problemas más generales donde no se conocen soluciones explícitas.

\section{REFERENCIAS}

[1] Arcoya, D.; Carmona, J. y Martínez-Aparicio, P. (2013). Radial solutions for a Gelfand type quasilinear elliptic problem with quadratic gradient terms. Recent Trends in Nonlinear PDEs II Contemporary Mathematics, 21-30.

[2] Ascher, U. M. (1995). Numerical solution of boundary value problems for ordinary differential equations. SIAM.

[3] Bebernes, J. y Eberly, D. (1989). Mathematical problems from combustion theory. Springer-Verlag.

[4] Bratu, G. (1914). Sur les équations intégrales non linéaires. Bulletin de la Société Mathématique de France, 42, 113-142.

[5] Castillo, P. (2002). Performance of discontinuous Galerkin methods for elliptic PDEs. SIAM J. Sci. Comput., 24(2), 524-547.

[6] Castillo, P. (2010). Stencil reduction algorithms for the Local Discontinuous Galerkin method. Internat. J. Numer. Methods Engrg., 81(12), 1475-1491.

[7] Castillo, P.; Cockburn, B.; Perugia, I. y Schötzau, D. (2000). An a priori error analysis of the Local Discontinuous Galerkin method for elliptic problems. SIAM J. Num. Anal., 38(5), 1676-1706.

[8] Castillo, P. y Sequeira, F. (2013). Computational aspects of the Local Discontinuous Galerkin method on unstructured grids in three dimensions. Mathematical and Computer Modelling, 57(9 10), 2279-2288. 
[9] Cockburn, B.; Kanschat, G.; Perugia, I. y Schötzau, D. (2001). Superconvergence of the Local Discontinuous Galerkin method for elliptic problems on Cartesian grids. SIAM J. Num. Anal., 39(1), 264285.

[10] Cockburn, B. y Shu, C. (1998). The Local Discontinuous Galerkin method for time-dependent convection-diffusion systems. SIAM J. Num. Anal., $35(6), 2440-2463$.

[11] Gelfand, I. (1963). Some problems in the theory of quasilinear equations. Amer. Math. Soc. Transl, $29(2), 295-381$.

[12] Gidaspow, D. y Baker, B. S. (1973). A Model for Discharge of Storage Batteries. Journal of The Electrochemical Society, 120(8), 1005.

[13] Jacobsen, J. y Schmitt, K. (2002). The LiouvilleBratu-Gelfand problem for radial operators. Journal of Differential Equations, 184(1), 283-298.

[14] Jalilian, R. (2010). Non-polynomial spline method for solving Bratu's problem. Computer Physics Communications, 181(11), 1868-1872.

[15] Lin, Y.; Bao, L. y Jia, X. (2010). Convergence analysis of a variant of the Newton method for solving nonlinear equations. Comput. Math. Appl., 59(6), 2121-2127.

[16] M. Scott, M. (1974). Conversion of boundary-value problems into stable initial-value problems via several invariant imbedding algorithms. Informe Técnico, Sandia Labs., Albuquerque, N. Mex.(USA).

[17] Markin, V. S.; Chernenko, A. A.; Chizmadehev, Y. A. y Chirkov, Y. G. (1966). Aspects of the theory of gas porous electrodes, in Fuel Cells: Their Electrochemical Kinetics. Consultants Bureau, 22-33.

[18] Mickens, R. E. (2005). Advances in the Applications of Nonstandard Finite Diffference Schemes. World Scientific.

[19] Perugia, I. y Schötzau, D. (2002). An hp-analysis of the Local Discontinuous Galerkin method for diffusion problems. J. Scientific Computing., 17(1-4), $561-571$.

[20] Roberts, S. y Shipman, J. (1976). On the closed form solution of Troesch's problem. J. Comput. Phys., 21(3), 291-304.

[21] Troesch, B. (1976). A simple approach to a sensitive two-point boundary value problem. J. Comput. Phys., 21(3), 279-290.

[22] Wanfl, Y.; Guo, Q. y PanflC, N. (2004). Thermoelectro-hydrodynamic model for electrospinning process. International J. of Nonlinear Sciences and $\mathrm{Nu}$ merical Simulation, 5-8.

[23] Weibel, E. S. (1959). On the Confinement of a Plasma by Magnetostatic Fields. Physics of Fluids, 2(1), 52 . 